\title{
Enquête sur la jeunesse étudiante de Le Play
}

Apprentissage scolaire et imprégnation saint-simonienne (1824-1832)

The young student Le play: An inquiry on his academic training and his saintsimonian impregnation (1824-1832)

\section{Antoine Savoye}

\section{(2)enEdition \\ Journals}

Édition électronique

URL : http://journals.openedition.org/ress/3929

DOI : $10.4000 /$ ress.3929

ISBN : 1663-4446

ISSN : 1663-4446

Éditeur

Librairie Droz

\section{Édition imprimée}

Date de publication : 15 décembre 2017

Pagination : 171-209

ISSN : 0048-8046

Référence électronique

Antoine Savoye, "Enquête sur la jeunesse étudiante de Le Play », Revue européenne des sciences sociales [En ligne], 55-2 | 2017, mis en ligne le 15 décembre 2020, consulté le 04 janvier 2021. URL http://journals.openedition.org/ress/3929; DOI : https://doi.org/10.4000/ress.3929 


\title{
ENQUÊTE SUR LA JEUNESSE ÉTUDIANTE DE LE PLAY \\ APPRENTISSAGE SCOLAIRE \\ ET IMPRÉGNATION SAINT-SIMONIENNE (I824-I832)
}

\author{
ANTOINE SAVOYE \\ Université Paris-8 Vincennes-Saint-Denis, CIRCEFT \\ asavoye@univ-paris8.fr
}

\begin{abstract}
Résumé. Pierre Guillaume Frédéric Le Play (1806-1882), après une période d'oubli, a retrouvé sa place au panthéon des sciences sociales. L'originalité de sa méthode d'enquête, formalisée dans les monographies de familles ouvrières, est reconnue. Les applications de cette méthode, soit par Le Play, soit par ses élèves, ont donné lieu à des ré-évaluations critiques, ouvrant la voie à une actualisation possible. Dans l'attente d'une biographie générale à la mesure du personnage, des vues d'angle sur différents moments de son existence viennent nous éclairer sur les conditions et les circonstances qui ont présidé aux élaborations méthodologiques et théoriques de l'auteur des Ouvriers européens (1855). Dans une perspective de mise en contexte biographique de l'œuvre de Le Play, nous nous proposons ici de revenir sur sa formation intellectuelle initiale alors qu'il est encore étudiant à Paris à la veille de son entrée dans la carrière d'ingénieur. II en ressort un portrait rectifié du jeune Le Play qui permet de mieux saisir comment il s'est confronté aux idées nouvelles, en particulier au saint-simonisme, sans jamais les adopter tout à fait, mûrissant une œuvre personnelle et originale.
\end{abstract}

Mots-clés: école des mines, enseignement supérieur, jeunesse, Le Play, saint-simonisme.

\begin{abstract}
After having been consigned to oblivion, Pierre Guillaume Frédéric Le Play (1806-1882) is finally considered one of the pionieers of social science. The originality of his case-study method, as elaborated in his research monographs on workers' families is widely recognized. Applications of this method, either by Le Play himself or by his disciples, gave rise to some critical reappraisals which pave the way for a possible actualization. Pending a final general biography which still need to be written, I will focus on some key moments of the life of the author of Ouvriers européens that shaped his methodological and theoretical production. In the prospect of a biographic contextualization of Le Play's work, I shall recount his original intellectual formation, whilst still a student in Paris, just before starting his career as a engineer. As a result, I bring out a refined portay of the young Le Play that enables us to better grasp how he confronted new ideas, particularly saint-simonism, without explicitly endorsing them, thereby giving birth to very personal and original works.
\end{abstract}

Keywords: graduate studies, Le Play, saint-simonism, school of mines, youth. 
«La biographie d’Auguste Comte, c'est la vie de Comte romancée par Comte et déromancée par l'histoire» Henri Gouhier, avant-propos, Vie d'Auguste Comte (1997, p. 15)

\section{AU COEUR DU QUARTIER LATIN}

Poursuivre des études supérieures après un baccalauréat obtenu à Rouen en I822 signifie pour Le Play retrouver Paris qu'il avait connu enfant à la fin de l'Empire. L'ambiance n'y est plus la même, surtout pour un jeune homme - il va sur ses I8 ans - qui s'installe au Quartier latin. On y débat en permanence du régime politique et des systèmes philosophiques et religieux. Les libéraux, partisans de la charte, s'opposent aux représentants d'un pouvoir qui incline vers une monarchie de plus en plus autoritaire. La religion est moquée par une partie de la jeunesse qu'on dit voltairienne à l'instar de ses aînés. Les souvenirs de la Révolution et de l'Empire comme ceux de l'Ancien régime agitent les esprits (Caron, I99I ; Goblot, 1995). C'est dans ce climat effervescent que se trouve plongé le jeune Havrais plus enclin aux promenades solitaires sur «le rivage maritime, les champs, les prés et les bois» (Le Play, 1989, p. 27) et aux excursions avec les pêcheurscôtiers quaux débats publics. Situation à haut risque pour un jeune homme sans fortune, loin de sa famille, et dont la destinée dépend de sa réussite scolaire.

\section{I.I.LE COLLÈGE ROYAL SAINT-LOUIS ET LA PRÉPARATION À POLYTECHNIQUE}

En janvier 1824, après un stage auprès de Jacques Dan de La Vauterie, un ingénieur des ponts et chaussées, ami de la famille, en poste à Valognes (Manche), Le Play fait une rentrée scolaire tardive, l’année étant largement commencée, au collège royal Saint-Louis. En intégrant la classe de mathématiques spéciales, c'est l'admission à Polytechnique que Le Play ambitionne. Cette orientation correspond à son goût pour l'étude positive des réalités concrètes : n’a-t-il pas envisagé de reprendre l'activité d'un géomètre-arpenteur du Havre? Elle s'explique aussi par la recherche de la sécurité matérielle. Polytechnique, c'est la perspective d'être admis dans un corps d'État avec la protection qu'une telle fonction implique. C'est se conformer au modèle de sa 
famille paternelle où l'on fait carrière dans l'administration: son grand-père fut agent des contributions indirectes et son père est officier des douanes. De ces deux années de classe préparatoire, Le Play ne dit presque rien dans le récit de sa jeunesse. Pourquoi un établissement parisien et non le lycée de Rouen? Sans doute pour mettre toutes les chances de son côté : près des deux tiers des candidats admis à Polytechnique sortent de Louis-le-Grand, Saint-Louis ou Charlemagne. Le collège Saint-Louis est un établissement flambant neuf, créé dans l'ancien collège d'Harcourt par une ordonnance de I8I7 (Bouquet, I89I). Que ses premiers proviseurs soient des ecclésiastiques n’en fait pas un établissement confessionnel déguisé. L’abbé Valentin Ganser qui vient de succéder à l'abbé Thibault, est d'origine allemande. Il a été longtemps maître de pension à Paris où il a eu Balzac comme élève. Il est décrit, par le biographe de Charles Gay', comme un «homme doux, grave, recueilli, paternel avec ses élèves» (du Boisrouvray, I92I, p. 8). Aidé de l'aumônier, l'abbé Dumarsais, il voudrait inculquer la piété aux élèves, mais c'est peine perdue. L’ambiance, parmi la jeunesse, est à la déchristianisation et les collèges sont, selon l'expression de Lamennais, en I819, des «séminaires d’athéisme » et des «vestibules de l'enfer». Peut-être cela est-il moins marqué à Saint-Louis quailleurs car les fils de famille nobles et catholiques y sont plus nombreux qu’à Louis-le-Grand, Henri-IV ou Charlemagne (Biré, 1904, p. 43). Lors de sa première année scolaire (amputée de trois mois), Le Play est externe. Sa mère étant au Havre et son père, officier des douanes, en poste en Moselle², peut-être loge-t-il chez une tante de sa mère, Marie Nicole Prévost ${ }^{3}$, afin de ne pas être abandonné à lui-même. À la distribution des prix, il obtient

I Charles Gay, futur évêque, entra au collège Saint-Louis en décembre 1823.

2 Pierre-Antoine Le Play et Rosalie (née Auxilion) vivent séparés depuis une dizaine d'années. Pierre-Antoine décède le 7 octobre 1854 à l'âge de 79 ans à Sarreguemines (Moselle) (d'après un extrait des archives de l'état-civil de Sarreguemines aimablement communiqué par M.D. Hemmert, archiviste municipal).

3 Marie Nicole Scholastique Prévost (née Auxilion en 1765) est une sœur de Louis François Auxilion, grand-père maternel de Frédéric. Elle a épousé Florent Germain Prévost, bonnetier près du jardin du Luxembourg à Paris. Veuve depuis 1818, elle a trois fils (dont deux travaillent au Muséum d'histoire naturelle) qui sont donc les cousins germains de Rosalie Le Play; ils se révèleront, toute leur vie, des appuis de Frédéric. 
le $3^{\mathrm{e}}$ accessit en physique, signe de grands progrès dans cette matière où il avait été en difficulté au baccalauréat ${ }^{4}$. L'année suivante, il est pensionnaire à la nouvelle Institution Mayer, fondée par un ancien élève de l'École polytechnique, Mathias Mayer d'Almbert ${ }^{5}$, et située rue Saint-Jacques, où il bénéficie, en sus des cours à Saint-Louis, d'une préparation au concours. Bruno Belhoste souligne le rôle de ce type d'institution : «Si certains élèves sont externes libres ou pensionnaires au lycée - c’est le cas de Galois -, la plupart, venus de province, sont en pension dans des établissements privés. » (Belhoste, 200ı, p. I 8). Les pensionnaires y sont entraînés à l'examen, encadrés par des professeurs de lycée, des répétiteurs de l’École polytechnique et des maîtres de mathématiques spécialisés. Ajoutons que l’Institution Mayer est réputée pour l'humanité de son directeur. «Ce n’était pas seulement un habile et savant professeur, communiquant sa science et son talent, c'était encore un ami affectueux s'attachant à former l'âme et le cœur en même temps qu'il faisait grandir l'esprit de ses élèves » écrit l'un de ses biographes à son décès. On ne sait rien des amitiés que Le Play a pu se faire parmi les élèves de mathématiques spéciales durant ces deux années. Il en retrouvera certains à Polytechnique: Jean Reynaud et Alphonse Gratry dont nous reparlerons, Léon Juchault de la Moricière ${ }^{6}$, Louis-Victor Duval, futur directeur d'une usine métallurgique dans la Drôme, Joseph Liouville, futur académicien des sciences, et Alphonse Trit, futur ingénieur des ponts et chaussées. Ces trois derniers, avec Gratry, sont admis la même année que lui et donc de la même promotion.

Durant sa seconde année scolaire (I824-I825), Le Play ne semble pas s'être consacré exclusivement à ses études. Il mentionne ses discussions passionnées avec deux étudiants allemands, pensionnaires comme lui à l’Institution Mayer, sur l'idée rousseauiste de la perfection originelle. Cela ne l'empêche pas d’obtenir le premier prix de physique et le second prix d'algèbre, devançant

4 Son professeur est Jacques Babinet (1794-1872), polytechnicien, spécialiste de l'électricité et futur astronome.

5 Mathias Mayer d'Almbert (1778-|84I) crée son institution après une carrière militaire dans les armées impériales dont il démissionne à la chute de l'Empire.

6 Léon Juchault de la Moricière (1806-1865) s'illustrera en Algérie avant d'entamer une carrière politique. II aura des sympathies saint-simoniennes. 
Liouville, autre pensionnaire de l'Institution Mayer7. Son maître en mathématiques est Louis Lefébure de Fourcy ${ }^{8}$ avec qui il va nouer des relations durables. Surtout, il est admis à l'École polytechnique. Les épreuves qui ont eu lieu au mois d'août, sont faites d'interrogations en mathématiques (arithmétique, algèbre, géométrie élémentaire, équations du premier et du second degrés, résolutions de triangles) et physique (statique appliquée à l'équilibre des machines), ainsi que d'une traduction d'auteurs latins, une composition française et la copie d'un dessin'. Le 2 I septembre i825, Le Play fait son entrée à Polytechnique où il bénéficie d'une demi-bourse sur les fonds du ministère de l'Intérieur, alors administration de tutelle, laquelle sera continuée en $1826-1827^{\circ}$. Le registre matricule de l’École le décrit comme un individu d's, 65 m, au visage ovale, avec un nez aquilin, un front découvert, une petite bouche et un menton rond, aux yeux et aux cheveux châtains.

\section{I.2. UN RAPPORT AMBIVALENT À POLYTECHNIQUE}

L'École polytechnique où Le Play est admis vient de faire l'objet d'une mise au pas $^{11}$. La royauté restaurée cherche, en effet, à y faire prévaloir ses valeurs contre les idées républicaines et l'attachement au souvenir de l'Empereur qui y subsistent encore. Pour contrer l’athéisme régnant, les élèves sont tenus de se

7 Joseph Liouville (1809-1882) que Le Play retrouvera à Polytechnique et, bien plus tard, en 1848, à la Haute Commission des études scientifiques et littéraires chargée, par le Gouvernement provisoire, de penser les réformes de l'enseignement supérieur.

8 Louis-Etienne Lefébure de Fourcy (1787-1869), polytechnicien, docteur es-sciences (I8II), est, en 1825, professeur de mathématiques au Collège Saint Louis et répétiteur de géométrie descriptive à Polytechnique. II a une solide réputation pédagogique de sévérité et de rigueur. Savant reconnu, il sera nommé à la chaire de calcul différentiel et intégral de la faculté des sciences de Paris (1843) et prendra sa retraite en 1863. Devenu proche des Le Play, il est l'un des témoins de Frédéric lors de son mariage (1837).

9 D'après «Programme des connaissances exigées pour l'admission à l'École polytechnique (Fourcy, 1828, p. 375).

10 Les frais de scolarité sont élevés: 1000 francs par an, en sus des frais d'uniforme et de matériel scolaire. On compte 19 boursiers ou demi-boursiers dans la promotion de Le Play, dont Gratry et Liouville.

II Réorganisée le 4 septembre 1816, puis remilitarisée partiellement en 1822, l'École a, à partir de cette date, deux militaires à sa tête. Ses élèves peuvent bénéficier de bourses relevant de trois ministères: I'Intérieur (8), la Guerre (12), la Marine (4). 
conformer à la religion catholique. Malgré cela, les idées philosophiques et politiques les plus avancées continuent d'y trouver un écho. Sur le plan des études, le programme évolue également. Sous l'influence de Laplace, le vieux rival de Monge, les cours de science appliquée sont supprimés au profit des disciplines théoriques, tandis que des cours de littérature et d'histoire apparaissent. Le régime disciplinaire est strict et Le Play en souffre : «Le souvenir de la souffrance que mavait causée le régime de casernement militaire, après les habitudes de liberté contractées depuis ma plus tendre enfance, ne s’est jamais effacé dans mon esprit» (Le Play, I989, p. 402, n. I). Les passions politiques propres à la jeunesse des écoles ne compensent pas cette souffrance car il y serait resté indifférent :

Par goût et par devoir, j'évitai la compagnie des camarades animés par des aspirations d'où sortit bientôt la révolution de I8zo. Soumis aux idées de hiérarchie, je me contentai de gémir intérieurement en entendant le professeur d'histoire glorifier le triste mot de Louis XIV «L'État, c'est moi». Je ne me laissai pas même éblouir par les leçons du Collège de France, où des professeurs éloquents discréditaient les coutumes nationales et signalaient «le développement de l'esprit humain» comme le but suprême de «la civilisation» (Le Play, 1989, p. 33).

En citant le Collège de France, Le Play vise Daunou dont le cours, à la chaire d'histoire et morale, est très suivi ${ }^{12}$. Sa critique épargne les cours en Sorbonne de Guizot (histoire et morale), Cousin (philosophie) et Villemain (littérature) où l'auditoire est encore plus nombreux. Ces cours publics nourrissent les débats qui mettent aux prises différents courants d'opinion entre lesquels se partage la jeunesse des écoles. Aux jeunes libéraux du Globe, emmenés par Théodore Jouffroy et Philippe Damiron, s'opposent les représentants du républicanisme jacobiniste et babouviste d'où sortira Étienne Cabet et son communisme. Le saint-simonisme, autour du journal Le Producteur (I825), est lui aussi en gestation, tout comme le fouriérisme. Le catholicisme n'est pas en reste et connaît un renouveau avec Le Correspondant (I829) de Louis de Carné auquel le tout jeune Charles de Montalembert collabore en attendant le lance-

12 Pierre Daunou (I76|-1840), à l'origine de la publication posthume de l'Esquisse d'un tableau historique des progrès de l'esprit humain de Condorcet, défend l'idée du «progrès des civilisations» et de leur perfectibilité indéfinie. 
ment de L’Avenir (1830 ${ }^{13}$. Enfin, la philanthropie, plus pratique mais non dénuée d'arrière-pensées politiques, offre une autre forme d'engagement qu'encourage François Guizot à la tête de la Société de morale chrétienne. Le Play, s'il ne s'est pas mêlé directement à ces débats, les a forcément connus.

Pour l'heure, il se tient sur la réserve et ses «deux premiers compagnons de prédilection » auraient été, comme lui, «étrangers à la politique » (Le Play, 1989, p. 33, n. 3). Il s'agit, tout d’abord, de Louis Robert ${ }^{14}$, un camarade connu au collège du Havre, que Le Play retrouve à Paris. Mais ils ne sont pas condisciples: quand Le Play est encore à Saint-Louis, Robert est déjà à Polytechnique qu'il quitte pour l'école spéciale de génie maritime de Brest au moment où Le Play y est admis. Les deux amis ne peuvent se voir que durant leurs moments de loisir et de liberté. Les rapports sont différents avec Gratry $^{15}$ qui, après Saint-Louis, est son condisciple à Polytechnique où il a été admis après une seule année de préparation. Elève brillant, doué pour les humanités - il a obtenu plusieurs distinctions au concours général ${ }^{16}-$, cette admission est pour lui aussi un choc:

D’abord le régime était dur. On travaillait depuis cinq heures et demie du matin jusqu'à deux heures et demie : c'est-à-dire neuf heures de travail continu, interrompu seulement à sept heures et demie par une récréation d'une demi-heure, pendant laquelle on mangeait un morceau de pain. Le premier repas était à deux heures et demie. Mais que travaillait-on? L'algèbre et tout ce qui s'y rapporte. Je l'avais bien prévu. C'était une effroyable privation et une révolution dans toutes mes facultés. [...] Tout donc autour de moi devenait sec, aride, géométrique (Gratry, 1925, p. 94-95).

13 Sur la fermentation des idées parmi la jeunesse à la fin de la Restauration, voir, outre Jean-Claude Caron (199I), François-André Isambert (1966).

14 Louis Robert dont le père était professeur d'hydrographie au Havre, accomplit une scolarité polytechnicienne très honorable. Faisant binôme avec Michel Chevalier, il passe à la Ire division en 1824, le 24e d'une liste de 94 élèves. Déclaré, l'année suivante, admissible dans les services publics, le $36^{\mathrm{e}}$ d'une liste de 95 élèves, il est admis à l'école d'application du Génie maritime le $2^{e}$ d'une liste de 4 élèves. Après avoir exercé comme ingénieur des constructions navales, à Cherbourg, durant dix ans (1827-1837), Robert bifurquera vers la prêtrise, tout en restant lié à Le Play qu'il soutiendra dans son entreprise de science sociale.

15 Alphonse Gratry, né le 30 mars 1805 à Lille, est son aîné d'un an.

16 Le second prix de dissertation latine et le premier de dissertation française en philosophie (1824). 
Le souvenir de Gratry demande, cependant, à être nuancé car l'enseignement comprenait également de la chimie (I3\% du temps total d'enseignement), de l'architecture et du dessin (20\%), ainsi que de l'histoire et des belles-lettres, il est vrai en portion congrue (6\%). À l'école de la Montagne Sainte-Geneviève, Gratry qui vient de se convertir au catholicisme ${ }^{17}$, déploie un grand zèle à «parler de religion et de vérité» à ses camarades dont, sans doute, Le Play. Ses résultats scolaires sont loin d'être ceux de son ami : il passe à la $\mathrm{I}^{\mathrm{re}}$ division en $\mathrm{I} 826$, le Iore d'une liste de I Io élèves. Il est déclaré, l'année suivante, admissible dans les services publics le $92^{\mathrm{e}}$ d'une liste de i I 3 élèves. Le registre matricule de l'École mentionne:

M. Gratry n’ayant pu, d’après son rang sur la liste générale par ordre de mérite, être classé dans l'un des services civils portés en sa demande, et une décision du ministre de la Guerre, en date du 3 octobre 1827 prise sur le rapport du comité de santé, l'ayant déclaré impropre au service militaire, S. Ex. le Ministre de l'Intérieur l'a autorisé le 12 novembre à passer une ze année de l'École. M. Gratry n'a pas profité de cette faveur et a donné sa démission. Il a cessé de faire partie des élèves à dater du ier novembre 1827.

Après cet échec, Gratry renonce à la carrière d'ingénieur et part rejoindre le petit cercle qui s'est constitué à Strasbourg autour du philosophe Bautain, devenu prêtre. Ordonné prêtre en I832, il deviendra une figure centrale de la renaissance de l'Oratoire ${ }^{18}$. Il est possible que la fréquentation de Robert et Gratry, jointe à celle d'un autre jeune Havrais, ami du premier, Pierre-Louis Labbé, venu à Paris faire des études de droit ${ }^{19}$, ait amené Le Play à se joindre au groupe d'étudiants catholiques qui gravitent autour de l'aumônier de l'École polytechnique, l’abbé

17 Auparavant, dira-t-il, «tous les prêtres continuaient à m'inspirer le plus profond mépris, et le langage dévot ordinaire le dégoût le plus insurmontable» (Gratry, 1925, p. 5I).

18 Gratry accomplira une double carrière dans l'enseignement (directeur du collège Stanislas, aumônier de l'École normale supérieure) et d'apologétiste. Élu membre l'Académie française en 1867, il participe à tous les débats touchant le catholicisme. Son amitié pour Le Play, comme son intérêt pour sa science sociale, ne se démentiront jamais.

19 Pierre-Louis Labbé, né en I80I, ancien élève du collège du Havre, sera ordonné prêtre en juin 1827. D'abord curé d'une petite paroisse rurale du pays de Caux, il rejoint bientôt son frère Léon-Xavier qui vient de fonder une maison d'éducation à Yvetot, celle-là même où enseignera Louis Robert. 
Martin de $\mathrm{Noirlieu}^{20}$. Ce dernier, prêtre intellectuel et mondain, était un personnage public auquel la famille royale avait confié l'éducation du duc de Bordeaux, petit-fils de Charles X, plus connu sous son titre de comte de Chambord.

L'image que Le Play forge rétrospectivement de ses années polytechniciennes souligne donc son acclimatation difficile au régime des études, mais aussi le décalage avec ses camarades de promotion. Ceux-ci, d'esprit frondeur, volontiers politisés, appartenant à des milieux plus fortunés, heurtent sa personnalité de jeune provincial d’origine modeste, concentré sur ses études et respectueux des autorités ${ }^{21}$. De fait, il ne semble pas avoir noué de relations durables avec beaucoup d'entre eux, comme l'indique le faible nombre collaborant, plus tard, à ses activités de science sociale. Ainsi, 30 ans plus tard, en I869, on ne relève, parmi les membres de la Société internationale des études pratiques d'économie sociale (SIEPES) qu'il a créée pour prolonger ses recherches des Ouvriers européens (1855), que les noms de Napoléon Daru et Léon Pavin de Lafarge ${ }^{22}$, alors que sa promotion comptait environ i 20 élèves. Gardons-nous, cependant, de conclusions hâtives relatives à un Le Play réfractaire au milieu polytechnicien (et réciproquement). Car d’autres faits indiquent qu'il s'y est mieux intégré qu'il veut le laisser croire. Tout d’abord, la correspondance adressée à sa mère lors de son premier voyage d'étude d'élève-mineur en 1829 témoigne de liens de groupe meilleurs que ceux suggérés 50 ans plus

20 Louis-François Martin de Noirlieu (1792-1870), gallican et mennaisien convaincu, aumônier à l'École polytechnique où il force, dit-on, le respect des élèves par sa tolérance et sa générosité. Choisi pour être sous-précepteur du duc de Bordeaux, il donne sa démission en novembre 1826. Le Play a donc pu le connaître comme aumônier au moins en 1825-1826 (sa Ire année). Également homme de lettres, il est l'auteur d'une réfutation, en 1823, des Ruines ou méditations sur les révolutions des Empires de Volney.

21 À l'École polytechnique, Le Play appartient, selon la statistique établie par Terry Shinn (1980) à partir du registre matricule de l'École, aux 13,6\% d'élèves issus des «fonctionnaires de niveau hiérarchique et officiers subalternes», recensés entre 1815 et 1829, catégorie qui, durant les 50 années suivantes, va aller s'amenuisant au profit des «rentiers et propriétaires» (déjà dominante avec 26,5\%) et des «professions libérales».

22 À ces deux noms, s'ajoute Louis de Kergorlay (né en 1804), très proche ami de Tocqueville, élève en même temps que Le Play, mais d'une autre promotion (1824) qui présidera la SIEPES. Ainsi que Michel Chevalier (promotion 1823) mais qui paraît n'avoir fait la connaissance de Le Play qu'une fois aux Mines. 
$\operatorname{tard}^{23}$. Par ailleurs, il est apprécié de plusieurs membres du corps professoral et non des moindres: François Arago, professeur de géométrie descriptive, Louis Thénard, professeur de chimie, Jean-Baptiste Dumas, alors répétiteur, qui seront ses protecteurs dès la sortie de l'École. Et lorsqu'en I848, il participe à la mise sur pied de l'éphémère École d’administration, c'est bien le modèle de formation polytechnicien qu'il tente de reproduire. Ce tropisme polytechnicien de Le Play explique aussi l'audience que rencontrera sa science sociale, de son vivant, auprès d'élèves de promotions postérieures à la sienne tels que Ildephonse Favé (I830), Achille Delesse (I837), Jules Michel (1848), Émile Cheyson (1854) ou Alexis Delaire (1856) ${ }^{24}$. En dépit d'un manque d'affinité certain avec le régime de l'École et d’un déficit relationnel au sein de sa promotion, Le Play n’en endosse pas moins l'éthos polytechnicien.

\section{UN ÉLÈVE-INGÉNIEUR À LA DÉCOUVERTE DU MONDE SOCIAL}

Passé en $\mathrm{I}^{\text {re }}$ division à la rentrée de $\mathrm{I} 826,2^{\mathrm{e}}$ sur I Io, puis, classé l'année suivante, $4^{\mathrm{e}}$ sur $\mathrm{I}$ I9, Le Play entre en $\mathrm{I}^{\mathrm{er}}$ rang à l'École des mines le 15 septembre I $827^{25}$. Pourquoi n’a-t-il pas opté pour les Ponts et Chaussées, école dont est issu son mentor, Dan de La Vauterie? Sans doute en raison de son goût pour la chimie qu'il peut mieux satisfaire aux Mines. Il y retrouve, admis en même temps que lui, Napoléon Garella, Henri de Grouchy, petit neveu du maréchal, et Charles Reverchon. Nulle part, Le Play ne les mentionne. Ils avaient été précé-

23 De passage à Metz au début de son voyage d'instruction, il écrità sa mère: «C'est à Metz que se trouve la plus grande partie de la promotion de l'École polytechnique. Elle compte environ 60 élèves à l'école d'application militaire. Dès que mon arrivée a été connue, j'ai eu nombreuse société et j'ai été immédiatement invité à partager l'ordinaire de la brigade composée pour la majeure partie de mon ancienne salle d'étude de l'École polytechnique. J'ai passé une soirée très agréable en me trouvant réuni avec tous les camarades dans un café» (lettre du 10 juin 1829) (Le Play, I899, p. 31).

24 Sur les ingénieurs des mines continuateurs de Le Play, voir Savoye et Audren, 2008.

25 Au sortir de l'École polytechnique, selon leur vœu et leur rang de classement, les élèves intègrent une des écoles d'application: dans l'ordre des effectifs accueillis viennent d'abord l'École de l'artillerie de terre et du génie militaire (Metz) et celle des ponts et chaussées, puis l'École des mines, l'École du génie maritime (Brest, Lorient, Paris), l'École d'artillerie de marine (Lorient), l'École des ingénieurs géographes (supprimée en I83I) (Belhoste, 2003). 
dés en I826 par Jean-Martial Bineau (futur ministre des Travaux Publics de la République de I848), Celse Gervoy, Scipion Gras et Jean Reynaud.

\section{I. L'ÉCOLE DES MINES ET SA PÉDAGOGIE ACTIVE}

Le régime de l'École des mines le change du tout au tout ${ }^{26}$. Des effectifs réduits impliquant moins de promiscuité27, une discipline favorisant la liberté des élèves délivrés de l'internat et du casernement, des enseignements faisant la place aux sciences appliquées et aux recherches expérimentales, une pédagogie active incluant des voyages dits « d'instruction » caractérisent une formation qui le séduit immédiatement. Les examens annuels auxquels l'élève doit se soumettre tant qu'il n'a pas atteint le nombre de points requis dans chacune des matières (le «medium»), ont lieu en avril-mai. Au cours d'une période d'un mois environ, les élèves ingénieurs sont interrogés, par écrit et oralement, sur les différents cours de l'année formant l'«instruction théorique» (exploitation des mines, docimasie, minéralurgie, minéralogie et géologie). Il y a également des examens de dessin et de langues étrangères, allemand et anglais, lesquelles, toutefois, sont facultatives. L'«instruction pratique» est constituée, d'une part, d'exercices ayant lieu durant le séjour des élèves à l'École (manipulations docimastiques, travaux géodésiques, levées de plan) et, d'autre part, de deux «voyages d'instruction» (dits aussi «campagnes d'été»), effectués en «brigade» de deux ou trois élèves, avec des objectifs précis et selon un itinéraire et un calendrier approuvés par le conseil de l'École. Ces voyages, d'une durée moyenne de 140 jours, sont à effectuer à la fin de la $2^{\mathrm{e}}$ et de la $3^{\mathrm{e}}$ années de la scolarité. Ils interviennent après la session des examens théoriques et ont pour but la visite de régions minières et métallurgiques et des établissements industriels qui s’y trouvent. Ils donnent lieu à la tenue d'un journal de voyage où sont consignées toutes les observations faites au cours de ce long déplacement, ainsi qu’à la rédaction de deux mémoires portant sur une question

26 Le régime de l'École est l'externat. Le Play loge à l'hôtel du Luxembourg, rue Saint-Dominique d'Enfer (aujourd'hui Royer-Collard), donc à deux pas de l'École.

27 L'École compte, en moyenne, une quinzaine d'élèves ingénieurs, toutes années confondues, et une douzaine d'élèves externes. 
définie au départ. Ces écrits sont à remettre au retour, dans un délai de quelques semaines que les élèves ont beaucoup de mal à respecter.

À l'École des mines, Le Play dit s'être rapproché de Reynaud admis l'année précédente. Ce dernier, provincial lui aussi, a fait ses études secondaires à Thionville, puis à Metz, avant de venir à Paris ${ }^{28}$. Bien que de caractère et de sensibilité très différents, nous dit Le Play, ils partagent tous deux le même «amour de la vie rurale et certaines vues d'intérêt public ». On pourrait y ajouter quelques analogies dans leur histoire personnelle: l'absence du père, contrebalancée par la forte présence de la mère et l'influence décisive d'un maître rencontré à l'adolescence. Merlin (de Thionville), l'ancien conventionnel régicide, devenu le tuteur de Reynaud, lui aurait appris, disent ses biographes, «l'amour de la patrie et de la république » et l'aurait familiarisé avec l'histoire de la Révolution. Le Play préfère insister sur le rôle de la mère de son ami qui lui aurait inculqué «ses éminentes vertus privées «(Le Play, I989, p. 35, n. I). Tout en reconnaissant l'ascendant de l'aîné sur le «conscrit» qu'il était, Le Play peut avec Reynaud se livrer à des discussions sur les questions sociales qu'il avait évitées jusque-là. Il en naît une solide amitié, mais aussi le constat d'un désaccord. À Reynaud qui commence «à se passionner pour les idées saint-simoniennes » et espère en une «transformation pacifique des rapports sociaux », Le Play oppose des opinions forgées au cours de sa prime éducation. À l'en croire, ses convictions n'auraient pas été entamées durant les années passées à Saint-Louis, puis à Polytechnique. Ces divergences, loin de séparer les deux élèves ingénieurs, les rapprochent au point d'envisager une confrontation concrète au cours d'un voyage d'instruction effectué de concert:

Nos discussions continuelles ne diminuèrent point le dissentiment qui régnait entre nous; mais elles accrurent notre affection, en nous donnant le désir d'une association qui pût nous conduire à la découverte de la vérité. En cette matière, sans s'attacher autant que moi à la méthode d'observation, Reynaud ne repoussait pas la pensée de prendre pour critérium de nos jugements les faits sociaux observés dans un voyage fait en commun (Le Play, 1989, p. 35). 


\subsection{LE HARTZ, PREMIÈRE EXPÉRIENCE DE TERRAIN}

C'est au printemps de 1829 que Le Play et Reynaud forment, en vue de leur «voyage d'instruction», le projet d'un vaste périple en Allemagne du Nord dont le cœur serait un long séjour dans le Hartz, avec le dessein d’associer à l'étude des mines, des ressources énergétiques (bois et houille) et des usines de traitement des minerais, celle de questions économiques et sociales ${ }^{29}$. Pourquoi l'Allemagne? Le Play justifie ce choix par un souvenir d'enfance! Ce qu'un ami de son oncle lui avait dit de cette «patrie de la sagesse», il y a une vingtaine d'années, l'aurait déterminé à y aller voir de près. D’autres raisons sont plus professionnelles. Le Hartz, région montagneuse, pauvre au plan agricole, est réputée pour ses richesses minières (plomb, cuivre, argent et fer) et, plus encore, pour l'habilité avec laquelle ses mines sont exploitées. Or, elle reste encore mal connue des ingénieurs français. Certes, Antoine-Marie Héron de Villefosse qui, sous l'Empire, a dirigé ce district minier l'a décrit ${ }^{30}$, mais cela remonte à plusieurs années et, depuis, les visites d'élèves-ingénieurs ont été superficielle (Lamé et Clapeyron en I822) ou partielle (Perdonnet en I828). Par ailleurs, et cela concerne la dimension sociale qui motive Le Play et Reynaud, ces mines présentent l'originalité d’être administrées par la puissance publique. Une population de 50000 personnes vit directement ou indirectement de leur exploitation dans des conditions qui étonnent les contemporains ${ }^{31}$. Situation qui remonte à loin puisque Montesquieu, déjà, avait vanté le sort des mineurs

29 Ce voyage est, pour Reynaud, le second. II avait effectué le premier en 1828 en compagnie de Michel Chevalier et Jean-Martial Bineau. Un périple de 137 jours les avait emmenés en Suisse alémanique, puis en Alsace, visitant des salines, une cristallerie (Baccarat), des mines de fer, des carrières à chaux, des tourbières, des hauts fourneaux et des forges (Framont), ainsi qu'une fabrique d'indiennes (Mulhouse).

30 Antoine-Marie Héron de Villefosse (né en 1774), polytechnicien et ancien élève de l'École des mines, avait été nommé par Bonaparte commissaire du gouvernement à Clausthal (1803).

31 En 1822, les aspirants ingénieurs Lamé et Clapeyron qui ont précédé Le Play et Reynaud s'extasient devantles effets de cette administration: «À notre arrivée à Andréasberg, nous avons éprouvé un vif sentiment de joie en voyant l'air d'aisance et de bonheur qui règne sur le visage de ces laborieux montagnards; nous avons béni l'administration paternelle qui a rendu cher aux habitans [sic] de ces forêts un sol ingrat, que la nature semblait n'avoir destiné qu'à nourrir des bêtes sauvages» in «Précis d'une course dans le pays du Hartz», Annales des mines, I822, p. 29. 
du Hartz qui, hommes libres, vivaient heureux ${ }^{32}$. À ces raisons techniques et sociales de s'intéresser au Hartz s'ajoute l'attrait d'une région dont les légendes et le relief, avec en son centre le fabuleux Brocken, ont de quoi séduire des jeunes gens en mal de romantisme. Dans son condensé de leurs 6 mois d'exploration, de juin à novembre I829 (Le Play, 1989, p. 35-39), Le Play, tout à la généalogie idéalisée de sa méthode de science sociale, ne restitue pas les détails de ce voyage fondateur. Ils sont pourtant fort instructifs sur l'état d'esprit de Le Play qui apparaît captivé par son apprentissage mais, parfois, frondeur vis-à-vis des institutions et de leurs représentants. Tout d’abord, le projet de Le Play et Reynaud d'explorer l'Allemagne du Nord en 200 jours ne reçoit pas immédiatement l'assentiment du conseil de l'École. Celui-ci fixe aux deux jeunes gens un programme précis et contraignant dont la durée est ramenée aux 148 jours réglementaires et qui leur impose de visiter, outre l’Allemagne du Nord (Io7 jours), la France de l'Est et la Belgique ${ }^{33}$. De plus, la place du Hartz est minimisée et ne compte que pour un tiers dans la longue liste des sites géologiques, des carrières, des mines et des usines que Reynaud et Le Play doivent visiter sur le terrain allemand. Enfin, les sujets des mémoires qu’ils doivent remettre à l'issue de leur exploration, confirment ce rééquilibrage. Le Conseil leur assigne l'étude de deux régions industrielles, le Hartz, certes, pour la fabrication du plomb, du laiton et du zinc, mais aussi la région liégeoise pour l'extraction de la houille et le traitement du minerai de fer ${ }^{34}$. Heureusement pour les deux amis, Louis Becquey, directeur général des Ponts et Chaussées et des Mines, eu égard aux résultats scolaires exceptionnels de Le Play, intervient en leur faveur et approuve la durée de leur projet bien qu'il excède le «standard» ordinaire des voyages d'instruction. Ils pourront disposer de 200 jours

32 «[...] avant que le christianisme eût aboli en Europe la servitude civile, on regardait les travaux des mines comme si pénibles qu'on croyait qu'ils ne pouvaient être faits que par des esclaves ou des criminels. Mais on sait qu'aujourd'hui, les hommes qui y sont employés vivent heureux». Et de préciseren note: «On peut se faire instruire de ce qui se passe, à cet égard, dans les mines du Hartz dans la basse-Allemagne, et dans celles de Hongrie» (Montesquieu, 1748).

33 Voir École royale des mines, Procès verbaux du Conseil de l'Ecole, année I829, Ms, p. 244-247 (Bibliothèque de l'École des mines, Paris). Aimablement communiqués par Mme Marie-Noëlle Maisonneuve alors en charge du fonds ancien.

34 Les deux élèves doivent aussi rapporter des échantillons de minéraux pour les collections de l'École. 
et d'un supplément d’allocation en conséquence ${ }^{35}$. Mieux, il encourage leur intérêt pour l'Allemagne; il les met en contact avec des personnalités allemandes résidant à Paris et leur fournit des introductions auprès des administrateurs des mines du Hartz par l'entremise de Héron de Villefosse.

Reynaud et Le Play respecteront ce programme imposé, se permettant seulement quelques écarts de calendrier, ainsi qu'une excursion hors programme à Berlin, avec un crochet par la Baltique pour Reynaud qui n’a jamais vu la mer. Ils partent au tout début du mois de juin I829 de Paris (le 5 ou le 6) pour un voyage qui durera jusqu'en novembre. Leur itinéraire les fait passer par Saint-Dizier, Bar-le-Duc, Metz. Ils entrent en territoire germanique à Sarrebrück et sillonnent la rive gauche du Rhin, parcourant le Palatinat, poussant des pointes jusqu'à Mayence et Coblence. Puis, après un séjour de trois jours à Holzapfel (où se trouvent des usines de plomb) et de cinq à Siegen, chef lieu de 4000 habitants d'une zone de mines d'argent, de cuivre et de plomb, ils gagnent Gottingue par Cassel et se trouvent alors aux portes du Hartz. Nous sommes le 10 août. De là, ils s'installent à Goslar ${ }^{36}$ qui sera leur établissement principal pour l'exploration du Hartz qui va se prolonger jusqu'à la mi-octobre. Dans une lettre à sa mère, Le Play décrit sa journée type :

Ma vie dans ce moment est des plus régulières. Voici un abrégé de l'emploi de mon temps. À quatre heures et demie du matin, je prends une tasse de chocolat au clou de girofle, d'après la coutume du pays, puis je m'embarque à pied pour Oker où j'arrive à six heures, où j'étudie dans les mines la fabrication du zinc, du cuivre, du laiton, du plomb, de l'argent, de l'or, du soufre, etc. qui se trouvent rassemblés sur ce même point; cette étude est fatigante parce que je suis obligé d'être constamment avec les ouvriers et de causer avec eux; j'entends à présent assez bien l'allemand, mais celui des ouvriers est comme le patois par rapport au français et j’ai beaucoup de mal à comprendre [...] à midi, je vais dîner dans une espèce de cabaret où je mange du bout des dents du jambon

35 Cette question budgétaire est importante pour un étudiant peu fortuné comme Le Play. Aux Mines, il complète les allocations versées aux élèves par des «travaux littéraires et scientifiques» notamment en vue de son voyage d'instruction. Son ami Saint-Léger témoigne qu'il donnait des leçons particulières dont il a lui-même bénéficié.

36 Goslar, ville historique de 6500 habitants, située dans le royaume de Hanovre, est le siège de l'administration des mines. 
et du bœuf salé avec des groseilles fermentées dans le vinaigre [...]. Pour me dédommager, je fais comme l'ami Clemson, j'embrasse les deux servantes qui, comme toutes les femmes du Hartz, sont très bien et je retourne jusquau soir huit heures à mes fourneaux, puis je rentre à Goslar (Le Play, I899, p. 60)37.

S'étant trop attardés dans le Hartz, Reynaud et Le Play doivent mettre les bouchées doubles pour accomplir le reste de la mission. De nombreuses visites de mines, mais aussi d'usines métallurgiques et de transformation des métaux les attendent sur le chemin du retour: Minden et Bochum (houille), Iserlohn (zinc, laiton), Solingen (coutellerie), Cologne et Aix-la-Chapelle (fabriques d'aiguilles et d'épingles), auxquels le programme a même adjoint des usines textiles comme à Elberfeld. Parvenus en Belgique par Aix-la-Chapelle, ils étudient la mine de zinc de la Vieille-Montagne, avant d'écumer le triangle Liège-Namur-Dinant, multipliant les visites de mines de charbon et de forges. Enfin, ils rentrent en France par Givet et, via Mézières, Reims et Soissons, rejoignent Paris vers la fin novembre où ils doivent se mettre dare-dare à la rédaction des mémoires attendus pour la fin de l’année. Le Play doit remettre un premier mémoire «complet et détaillé sur la fabrication du zinc et du laiton au Hartz, à Iserlohn, à Stolberg, à Liège et à Givet», et un second sur «le gisement, l'exploitation et le traitement des minerais de fer, tant au charbon de bois quau coke dans les environs de Liège ». Quant à Reynaud, on attend de lui un mémoire «complet et détaillé sur une usine à plomb cuivreux et argentifère du Hartz» et un autre sur «le gisement de la houille à Liège et sur l'exploitation d'une mine en particulier ». Les mémoires de Le Play ne figurent pas, malheureusement, dans la collection des mémoires des élèves conservés à l'École des mines de Paris ${ }^{38}$. On y trouve, par contre, celui de Reynaud sur «la fabrication du plomb, du cuivre et de l’ar-

37 Ces anecdotes éclairent sur la vie affective de l'étudiant Le Play. II est loin d'être insensible aux charmes des jeunes filles croisées au cours de son voyage. Néanmoins, Albert de Saint-Léger prétend qu'«il n'avait jamais offensé la chasteté» (Baunard, 1902, p. 283). On peut en douter...

38 Seuls les carnets de notes de voyage de Le Play sont accessibles et consultables en ligne. Guy Thuillier a été un des premiers à appeler l'attention sur l'intérêt de ces mémoires (Thuillier, 1962). On trouve, notamment, dans la collection plusieurs mémoires de Michel Chevalier. Je remercie Mme Anne Weber, responsable du fonds patrimonial à la Bibliothèque de l'École des mines, d'avoir aimablement facilité mes recherches. 
gent à l'usine de $S^{t}$ Andreasberg ${ }^{39}$. En post-scriptum, Reynaud explique qu'il n'a pu réaliser le second mémoire, son état de santé l’ayant empêché de descendre dans les mines de houille du bassin de Liège, et sollicite l'indulgence du Conseil.

Le Play affirme que ce séjour dans le Hartz a été l'occasion de ses premières observations sociales (Le Play, 1989, p. 38). On peut, sans peine, le croire lorsqu'il dit avoir étudié les différentes catégories professionnelles (mineurs, fondeurs, chargeurs, manœuvres, etc.) cotoyées quotidiennement. Par contre, on est plus dubitatif lorsqu'il affirme que «cette étude fut menée de front» avec celle des populations rurales de la plaine saxonne et, surtout, que «ces recherches s'étendirent également aux pêcheurs côtiers du Hanovre, de l'Oldenbourg et des Pays-Bas ». En effet, à quel moment les deux amis auraient-ils été disponibles pour aller enquêter en des régions aussi éloignées de leur itinéraire? Certes, Reynaud est allé aux bords de la Baltique, probablement entre Lübeck et Rostock, où il a vu les populations maritimes, mais sans Le Play qui, resté dans le Hartz, y poursuivait ses travaux. Et, concernant les Pays-Bas et la région d’Oldenbourg, quand les deux élèves-ingénieurs auraient-ils eu le loisir de s'y rendre, alors que ces régions sont très au nord de la ligne Clausthal-Iserlohn-Cologne-Aix qu'ils ont suivie après avoir quitté le Hartz? On sait qu'ils se déplaçaient vite : «Dans notre ardeur voyageuse, pendant la dernière semaine, nous avons fait au moins I4 lieues par jour » écrit Le Play à sa mère de Clausthal, le ı octobre. Mais nous n'avons pas de trace (mention d'un lieu, d'une rencontre) confirmant un tel détour. La question des enquêtes effectivement menées reste donc ouverte, y compris la célèbre monographie du mineur du Hartz qui, 20 ans plus tard, fera l'objet d'un premier exposé devant la commission du Luxembourg présidée par Louis Blanc en mars I848. En effet, la version que Le Play en publie, en 1855 (Le Play, I855, p. I40-145) ne fait pas référence à des observations effectuées en I829, mais seulement lors sa deuxième visite de la région en I845, alors en compagnie d'Albert de Saint-Léger. Les observations de I829 ne sont mentionnées que dans la seconde édition remaniée de cette monographie (Le Play, I877, p. 99-I52). Celle-ci est complétée par une enquête sur la 
condition des familles rurales dans le Lunebourg dont on ignore si les données relèvent du premier ou du second voyage. Manifestement, Le Play a hésité sur le statut de ses toutes premières observations, celles de i829: dans la première édition des Ouvriers européens, il ne les juge pas dignes d'être mentionnées dans son magnum opus. Mais, une vingtaine d’années plus tard, en vue d’une généalogie de sa science sociale, il les intègre comme source de sa monographie dans sa deuxième version. Cela lui permet d'introduire un épisode non évoqué en I855: les conseils du directeur général des mines du Hartz, Julius Albert (ou Alberts) ${ }^{40}$, à l'«élève parisien» (sic) lui recommandant de visiter les foyers domestiques du Lunebourg hanovrien. Cette enquête a-t-elle eu lieu à cette époque? Il semble que non car il précise : «cet enseignement ne porta point d'abord tous ses fruits» (Le Play, I877, p. I48). Au soir de sa vie, concernant ses toutes premières enquêtes, celles qu'il fit encore étudiant, Le Play brouille donc les pistes plutôt qu'il les démêle. Qu’a-t-il vu et étudié exactement ? Il nous laisse dans l'incertitude. Il est plus convaincant lorsqu'il affirme que l'expérience de I829 lui a révélé la méthode d’enquête de terrain qu'il adoptera désormais et qu'il recommande à «la jeunesse des classes dirigeantes». À celle-ci, il faut enseigner l'art des voyages en perfectionnant le modèle en vigueur à l'École des mines comme Reynaud et lui l'ont fait empiriquement en I829 lorsqu'à l'étude des industries minières et métallurgiques, ils ont associé des enquêtes de caractère social, soit par des investigations directes auprès des populations, soit par des entretiens auprès des notabilités locales dont les pratiques ont été également observées. Le Play détaille les divers dispositifs et techniques de ce type d'enquête à double vocation, professionnelle et savante. L'ambiance qui préside à de tels voyages d'étude est également primordiale à ses yeux. Il insiste sur la relation qui l'a uni à Reynaud, préfigurant l'«esprit de sociabilité » qui, dit-il, lui permettra de «mener à bonne fin [...] beaucoup d'autres voyages avec des amis ayant presque toujours des caractères fort différents du mien» (Le Play, 1989, p. 39). Mais, à nouveau, Le Play simplifie la réalité de cette première expérience pour les besoins de sa démonstration. 
Il escamote la présence, durant tout le séjour dans le Hartz, d'un troisième homme, un étudiant américain, Thomas Clemson ${ }^{41}$. Dans une missive à sa mère, Le Play dépeint Clemson qu'il connaît depuis l'École, comme «le garçon le plus aimable et le plus amusant qu'il soit possible de voir; il a pris entre autres choses à tâche d'embrasser toutes les gardeuses de chèvres et de vaches que nous rencontrons dans les montagnes» (Le Play, I899, p. 57). Le couple Reynaud-Le Play qui voulait débattre de hautes questions sociales est devenu un trio de gais lurrons; «cette fois encore le fameux proverbe "plus on est de fous, etc." ne s'est pas trouvé en défaut» (op.cit., p. 52).

On trouve dans le récit de 1879 d'autres écarts avec ce que fut la réalité prosaïque telle que, dans l'instant, Le Play la rapporte à sa mère. Ainsi, lorsqu'il déclare péremptoirement: «Pendant sept mois nous sûmes, en dehors de toute excitation urbaine, trouver en nous-mêmes la source d'une complète activité de corps et d'esprit» (Le Play, I989, p. 39), il passe sous silence un déplacement à Berlin où une soirée à l'opéra et la visite du château de Sans-souci à Postdam l'enchantent. Il justifie cette incartade :

Ce petit voyage qui forme un petit accroc à l'itinéraire qui nous a été tracé m’est presque nécessaire dans la situation actuelle après un séjour prolongé dans des mines très malsaines. Ce travail un peu forcé ne laisse pas que de me fatiguer, j’ai été obligé, entre autres choses, de renoncer au vin pour quelques temps, parce que l'acide du vin, en se combinant avec les vapeurs de plomb, produit un poison qui occasionne de fortes coliques (Le Play, I899, p. 59).

4I On retrouve Clemson mentionné dans la correspondance de Chevalier à Le Play en 1834 et son nom apparait dans les Annales des mines (t. II, 1832) pour ses recherches sur la seybertite avec la mention "professeur de chimie". Thomas Green Clemson (1807-1888) est le fils d'un riche quaker de Philadelphie. Son intérêt pour la chimie l'amena, jeune homme, à Paris où il suivit des cours à la Sorbonne et fut admis à l'École des mines comme élève étranger. Diplômé de l'Ecole, il devint ingénieur conseil et poursuivit ses recherches en chimie des métaux et en géologie. À la suite de son mariage avec une fille de John C. Calhoun, homme politique important, deux fois vice-président des États-Unis (I824 et 1828), il supervisa les propriétés minières de son beau-père en Georgie. II revint à plusieurs reprises en Europe, notamment comme chargé d'affaires auprès du royaume de Belgique (1844). Pionnier de l'application de la chimie à l'agriculture, il développa l'enseignement technique, notamment en Caroline du Sud. Il est à l'origine de l'actuelle Clemson University. 
Il peut d'autant plus se le permettre qu'une lettre de Becquey, datée du i5 juin I829, vient de l'informer qu'il totalise au terme de sa deuxième année un nombre de points de mérite «auquel, depuis la fondation de l’École, n’a jamais atteint aucun élève, même de 4ème année» (Le Play, 1989, p. 404) ${ }^{42}$. Cela lui vaut d'être «hors de concours », ayant d'ores et déjà totalisé le nombre de points nécessaires pour être déclaré ingénieur, sous réserve, cependant, d’effectuer son second voyage d'instruction et de rendre les mémoires et les journaux attestant de la bonne réalisation des deux voyages exigés. Le Play est récompensé par des ouvrages dont les Voyages minéralogiques et géologiques de François Sulpice Beudant et la Richesse minérale de Héron de Villefosse. Il apprécie le geste avec humour confiant à sa mère: «Tu vois, ma chère maman, que notre honnête directeur ne me donne pas seulement du vent puisque l'ouvrage de M. de Villefosse, entre autres, coûte au moins 300 francs $\gg^{43}$.

\subsection{UN ACCIDENT PROVIDENTIEL?}

Sur la lancée de ce voyage, revenu à l'École, Le Play poursuit des études désormais dégagées de tout souci d'examen en dehors des deux mémoires à remettre pour la fin de l'année ${ }^{44}$. Il consacre une partie de son temps, durant l'hiver I829-1830, à enquêter, pour son compte, sur les industries parisiennes dont il visite les ateliers ${ }^{45}$. Mais, surtout, il conforte ses compétences en analyse chimique des substances minérales. Il se fait connaître des milieux savants en publiant deux analyses chimiques de minéraux, l'une de la tourmaline provenant

42 Déjà l'année précédente, le Conseil de l'École avait mentionné, dans une «note confidentielle», sur les élèves que l'«application» de Le Play était «très soutenue», que son «caractère» était «bon» et que sa «conduite» était «excellente» (procès-verbal du 13 mai 1828).

43 Le Play manifestera toujours de la considération pour Becquey (I760-1849), son premier chef. II a de l'estime pour sa personnalité d'homme public et de grand commis de l'État (voir Beugnot, 1852).

44 Pierre Berthier, le professeur de chimie, signale le 20 janvier 1830, que «M. Le Play achève en ce moment la rédaction du voyage qu'il a effectué pendant l'été dans le Hartz; la partie qu'il a déjà remise au conseil de l'École est faite avec le plus grand soin et enrichie d'une multitude de croquis qui équivalent à des dessins et qui donnent une idée de toutes les choses qu'il décrit».

45 Ces visites sur lesquelles il insiste restent mystérieuses: n'auraient-elles pas été aussi l'occasion de diffuser les idées saint-simoniennes auprès des ouvriers parisiens? 
de la morène du glacier Macugnana près du Mont-Rose, l'autre d'une substance associée à l'amphibole que l'on trouve dans les carrières de Saint-Yrieix (HauteVienne). Ses résultats concernant la tourmaline, très étudiée en raison de ses propriétés électriques, ont les honneurs des Annales de chimie et de physique publiées (Le Play, I829) sous les auspices de Gay-Lussac et Arago. Le Play, très sûr de lui, n’hésite pas à y contester la méthode d’analyse de l’Allemand Johann Friedrich Gmelin pourtant spécialiste du sujet. Le succès de ces études concourt à sa nomination au laboratoire de l'École des mines comme adjoint du professeur Pierre Berthier ${ }^{4}{ }^{6}$. Celui-ci, dans la lettre au Conseil de l'École où il soutient sa candidature, souligne que «M. Le Play a une aptitude égale pour toutes les sciences [...]. Il a un goût prononcé pour l'étude», mais aussi qu’il est «aimé de tous les élèves, grâce à ses talents et à la douceur de son caractère et qu'il exerce sur eux une influence qui tournera à leur profit». Il précise également que Le Play devra, d'une part, se consacrer à des recherches en chimie (consistant en l'analyse des échantillons adressés au laboratoire et des expériences docimastiques) et, d'autre part, s'occuper de l'instruction pratique de ses camarades $^{47}$. Cette candidature est approuvée par Becquey le 8 février i8zo. C'est dans ce laboratoire que, trois mois plus tard, il est victime d'un accident. Le 30 avril, lors d'une préparation de potassium, en compagnie d'un aide mis à sa disposition par le professeur Georges Sérullas du Val-de-Grâce, il est gravement brûlé aux deux mains. Dans la lettre qu’il adresse, le 2 mai I83o, au nouveau directeur général des Ponts et Chaussées et des Mines, Victor Legrand ${ }^{48}$, pour lui rapporter l'accident, Berthier en minimise les conséquences: «Vous savez

46 Pierre Berthier (|782-|86|), ancien élève de Polytechnique et ingénieur des mines, est professeur de docimasie et chef du laboratoire de l'École des mines depuis 1816, fonction qu'il conservera jusqu'en 1845. Personnalité scientifique de premier plan, ses travaux se répartissent entre la minéralogie, la géologie, la chimie, et intéressent autant les applications industrielles que la recherche fondamentale. II publie en 1834 un Traité des essais par la voie sèche, ou, des propriétés, de la composition et de l'essai des substances métalliques et des combustibles, ouvrage de référence pour les ingénieurs des mines.

47 L'accès au laboratoire est très convoité et Berthier doit alors faire face à une fronde des élèves externes qui lui reprochent de le limiter aux élèves ingénieurs issus de Polytechnique.

48 Victor Legrand (I79|-1848), ancien élève de l'École polytechnique (promotion 1809), ingénieur des Ponts et Chaussées (18|8), a succédé à Becquey. Le Play nouera des relations étroites avec lui. 
ce qui a été fait pour les secourir [Le Play et Fiquet] et la manière dont nous avons été secondés par M. le Docteur Coulomb et par M. Sérullas. M. Le Play a eu les deux mains entièrement dépouillées d'épiderme, mais, grâce à Dieu, il n'est atteint d'aucune blessure grave : il a souffert cruellement mais, maintenant, il est paisible et il va aussi bien que possible et demain ou après toutes les douleurs seront apaisées ». Et d'ajouter : «Il a tout supporté avec un courage et une force d'âme que l'on ne saurait trop admirer [...]. M. Le Play a eu pour consolation de voir combien il est aimé et estimé de ses camarades et de toutes ses connaissances $\gg^{49}$. En réalité, ses blessures s'avèrent plus sérieuses qu’il n’y paraît tout d’abord et, malgré les soins prodigués, il ne s'en remet pas aussi vite qu'imaginé par Berthier ${ }^{50}$. Conséquence immédiate : trois mois plus tard, c'est en spectateur impuissant et tourmenté qu'il assiste à la Révolution de juillet I8zo dans laquelle s'engagent nombre de ses camarades, à commencer par Michel Chevalier. Ce dernier qui a rejoint les saint-simoniens depuis une année environ, n'hésite pas à se mettre en avant. De concert avec Armand Bazard, il entreprend une démarche auprès de Lafayette afin que celui-ci proclame la dictature. Ce n'est pas seulement des Trois glorieuses dont Le Play est écarté, mais du bouillonnement politique et intellectuel qui suit les Journées de juillet ${ }^{51}$. Rétrospectivement, il interprète cette abstention forcée comme ayant, elle aussi, déterminé sa vocation sociale :

Privé de mes deux mains, réduit à une complète impuissance, je n’avais pour diversion à mes maux que la société des condisciples et des personnes compatissantes qui se pressaient autour de mon lit. [...] Ce dur apprentissage de la douleur, complété par une méditation forcée, m’apparaît aujourd’hui comme

49 Berthier à V. Legrand, lettre du 2 mai 1830, 3 p., AN FI427312.

50 Michel Chevalier dans une lettre de 1833 (Ms, fonds Le Play, Bibliothèque de l'Institut, Paris) évoque «le lit sur lequel vous êtes si longtemps et si douloureusement resté gisant dans le cabinet chinois». Et Eugène Lefébure de Fourcy précise: «On le transporta dans le cabinet chinois attenant à la salle du Conseil [...]; on y installa un lit provisoire [...] et, en attendant l'arrivée de sa mère et de sa soeur mandées du Havre en toute hâte, ses camarades se relayèrent tour à tour pour entretenir sur ses mains un courant d'eau froide, seul adoucissement à ses souffrances» (Le Play, 1899, p. 8).

5I Sur ce bouillonnement, voir Pierre Flottes (1927) qui restitue minutieusement le climat postrévolutionnaire dans lequel Alfred de Vigny, proche de Philippe Buchez, produit ses œuvres à thèse: La Maréchale d'Ancre (I83I), Paris (I83|) et Stello (1832). 
un des évènements décisifs de ma carrière : ce fut alors, en effet, que je pris la résolution de remédier autant que possible aux fléaux déchaînés dans mon pays (Le Play, I989, p. 40 et 4I).

Selon ses dires, il faudra à Le Play longtemps pour se remettre totalement ${ }^{52}$. Il affirme, en effet, n'avoir recouvré la plénitude de ses forces physiques quaprès I8 mois, c'est-à-dire vers la fin de I83 I, ou même «au milieu de l'année I832 » (Le Play, I989, p.406). De fait, ce grave accident a d’abord un impact sur le bon accomplissement de sa scolarité. En effet, bien qu’ayant été déclaré hors de concours, Le Play doit accomplir son deuxième voyage d'instruction. Or, dans l’immédiat, il n'est pas en état de le faire. Cette question occupe plusieurs séances du Conseil de l'École, partagé entre le respect du règlement, la prise en considération des séquelles de l'accident et la valeur exceptionnelle de l'élève ${ }^{53}$. Son enjeu n'est pas mince car elle implique le classement entre les élèves lequel détermine leur nomination dans le corps avec des effets à long terme sur leur avancement ultérieur. Concrètement, Le Play est en rivalité avec Gras et Gervoy lequels ont satisfait eux aussi au medium - avec un total de points nettement inférieur au sien - mais ont réalisé les deux voyages. Le Conseil, dans l'attente de l'effectuation du second voyage, décide de récompenser Le Play pour la qualité de son mémoire et de son journal de voyage de i829- qui «contiennent une masse considérable de documents et de dessins dont la plupart sont très précieux et [...] dignes d'être insérés dans les Annales des mines $\gg^{54}$. Puis, devant l'indisponibilité persistance de Le Play et considérant que «son voyage de i829 peut valoir deux campagnes », il décide de l'en dispenser et de le nommer aspirant devant ses condisciples Gras et Gervoy à dater du $\mathrm{I}^{\mathrm{er}}$ septembre $1830^{55}$, décision approuvée par le directeur général des Ponts et Chaussées et des Mines d’abord réticent.

52 D'après Eugène Lefébure de Fourcy, Le Play devrait sa guérison définitive à l'intervention de Guillaume Dupuytren, chirurgien en chef à l'Hôtel-Dieu, réputé pour sa science des brûlures.

53 Voir École royale des mines. Procès verbaux du Conseil de l'École, 18 et 19 mai 1830, p. 309, 29 juin 1830, p. 330 et 29 août 1830, p. 343.

54 Le Play reçoit un «baromètre de hauteur» et une «loupe à la Wollaston», prisme à décomposer la lumière inventé par l'Anglais Wollaston.

55 Notons que cette délibération a lieu le 29 août 1830 alors que, du fait du changement de régime, il n'existe plus alors qu'un «commissaire provisoire des ponts et chaussées des mines». 
Le Play est bien conscient d'avoir bénéficié des faveurs de la hiérarchie lorsqu'il écrit, avec son art de la litote: «Les dispositions bienveillantes qui inspiraient, en I829, le chef du corps des mines [Becquey] continuèrent chez ses successeurs, malgré l'ébranlement produit dans le gouvernement de la France par la révoution de I830. L'estime accordée à mes premiers succès fut conservée par le "dossier administratif” parmi les nouveaux gouvernants» (Le Play, 1989, p. 405-406).

$\mathrm{Si}$ un voyage d'instruction lui reste impossible, à la reprise des cours, le $\mathrm{I}^{\mathrm{er}}$ septembre 1830 , il reprend progressivement ses activités au laboratoire de l'École. Mais sa fonction d’adjoint savère très prenante sans être reluisante financièrement. Contrairement à ses camarades, il ne bénéficie pas d'indemnités de bureau ou de déplacement et n’a d’autre traitement que celui d’aspirant ingénieur d'un montant de 125 francs par mois. Dans une lettre du $\mathrm{I}^{\text {er }}$ juillet I83 I, il s'ouvre de ses difficultés matérielles au directeur général: «J’ai accepté avec reconnaissance cette mission honorable qui me permet de macquitter de mes devoirs envers la patrie dans le genre d’activité que j’affectionne le plus. Je viens vous prier maintenant, Monsieur le Directeur, de vouloir bien compléter cette faveur en me tirant de la position désavantageuse, sous le rapport pécuniaire, où me place cette carrière spéciale». Cette demande est appuyée par l'inspecteur des études qui souligne la lourdeur de la tâche qu’on lui a confiée: «Par la nature de la place, la surveillance des manipulations docimastiques, il est obligé d'être à l'École des mines depuis 8 heures du matin jusqu'à 6 heures du soir, et sans pouvoir sabsenter un seul jour $\gg^{56}$. Reconnaissant le bien fondé de la réclamation, le directeur général accorde à Le Play une indemnité de 800 francs qui sert sans doute à financer un voyage que, profitant des congés de l'École, s'octroie immédiatement notre convalescent. En compagnie d’un élève ingénieur des Ponts et Chaussées, Gustave Baumgarten ${ }^{57}$, de

56 Cette lettre et le voyage qui suivit confirment que Le Play est remis de son accident au cours du premier semestre de 1831.

57 Gustave Baumgarten (1808-1856). Fils de militaire, il est admis à Polytechnique en I827, puis aux Ponts et Chaussées en 1830. Au printemps de 1831, encore élève, il est envoyé au Havre pour y être employé aux travaux du port. Sa mission est appréciée par son supérieur qui précise dans son compte rendu du 28 novembre 1831 : «II [Baumgarten] a fait un voyage dans les ports de Bretagne avec monsieur Le Play, ingénieur des mines. Profitant des connaissances de son compagnon de voyage, il a recueilli des notes géologiques, en même temps qu'il observait les divers travaux des ports et qu'il les comparait avec ceux du Havre... » (AN FI427I3/I). 
deux ans son cadet, alors attaché aux travaux du port du Havre, il parcourt, durant l'automne de I83ı, le littoral normand et breton du Havre à Saint-Malo. Pourquoi le choix de Baumgarten? En raison de sa présence au Havre, patrie de Le Play où demeurent sa mère et sa soeur? Plus probablement parce que Baumgarten partage avec son aîné des sympathies saint-simoniennes. Le récit de leur voyage, muet sur ce plan, se présente sous forme d’un journal tenu par Le Play. Il débute par ces lignes, significatives de la renaissance du jeune homme:

Adieu, pour longtemps j’espère, la douleur et les médecins; échappé pour ainsi dire à l'autre monde, je pars pour faire encore une campagne dans celui-ci; ce n'est point dans le Jourdain mais dans les eaux de la vieille Armorique que je vais me régénérer et chercher une vigueur nouvelle; un jeune conscrit admirateur de la belle nature va partager mes dangers et mes travaux. Désireux d'aventures de tout genre, méprisant la fatigue, estimant la bonne chère, résignés à souffrir la mauvaise, le gousset assez bien fourni d'argent, nous partons avec tous les éléments possibles de succès. Que le dieu des voyageurs nous soit favorable (Le Play, sd, c. I8zI).

Au fil des pages, c'est surtout la géologie des pays traversés, la flore aussi, ainsi que les activités industrielles et économiques que décrit l’auteur. Avec, cependant, quelques digressions sur des incidents qui émaillent le voyage, comme l'arrestation par les gendarmes de Le Play qui, imprudemment, voyage sans passeport. Le passage à Granville est l'occasion, pour lui, de rappeller l'«héroïque défense» des Granvillais, l’an III de la République, face à l’armée vendéenne qui voulait faire du port un point de jonction avec la marine anglaise. Cette évocation des hauts faits révolutionnaires aurait pu être l'occasion d'une allusion à la famille maternelle de Le Play et à son grand-père granvillais engagé dans les armées républicaines. Mais il n’en dit rien. Par contre, ses remarques sur un monument élevé à Caen en souvenir de l'assassinat du duc de Berry, confirment ses inclinations politiques du moment :

[... en passant devant la place du collège, nous avons remarqué une colonne carrée en granite [sic] élévée, je crois, en expiation de l'assassinat du duc de Berry. Il est probable qu'autrefois, quelque bas-relief ou quelqu'inscription rappellaient les gémissements et les regrets de la France. Probablement aussi, lors de la glorieuse révolution de Juillet, le patriotisme des Caennais se sera vu obligé faire justice de toutes ces faussetés (ibid.). 
Témoignant du même état d'esprit, Le Play conte l'anecdote suivante recueillie lors de sa traversée pour Saint-Malo: «Le patron nous apprit qu'il avait transporté l'année précédente Polignac de St Malo à Granville où il se fit arrêter avec une stupidité digne de l'homme qui prétendait gouverner la France avec des ordonnances » (ibid.)

Il ironise également sur les rentiers retirés en province et élevés au rang d'électeurs par la récente loi, assise de la monarchie parlementaire orléaniste ${ }^{8}$. Évoquant Coutances (Manche) qu'il vient de visiter :

La ville a un certain air d'aisance; l'électeur à 200 fr. doit y abonder, je me trompe fort s'il n'y a pas un bataillon de garde nationale. C'est un de ces nombreux endroits où l'honnête marchand de la rue St Denis, en se retirant des affaires, peut aller jouir avec faste de ses 4 mille fr. de rente, et se condamner au repos en regrettant pour le reste de sa vie l'aune à laquelle il était accoutumé depuis trente ans (ibid.).

De retour à Paris, Le Play est nommé, le 25 octobre i83 I, ingénieur ordinaire de $2^{\mathrm{e}}$ classe $^{59}$. Il a 25 ans. Ici, se clôt la phase d'apprentissage scolaire qui l'a mené de la classe de mathématiques spéciales du collège Saint-Louis au laboratoire de l'École des mines. Si le voyage d'instruction dans le Hartz de I829 en a été incontestablement un temps fort, ses travaux de chimie comme de statistique ${ }^{60}$ ne doivent pas être négligés. Ces exercices revèlent sa capacité exceptionnelle à transcrire des faits en données numériques: les monographies de familles avec leur pièce centrale, le budget des ressources et dépenses annuelles, sont en germe dans ses travaux d'élève-ingénieur. Mise en regard

58 La loi électorale du 19 avril 1831 a élargi le corps électoral en abaissant le cens de la députation -calculé à partir des contributions directes versées au trésor public- à 200 frs pour être électeur et à 500 frs pour être éligible. Les premières élections eurent lieu le 5 juillet I83I. II est probable que Le Play qui remplissait la condition d'âge - 25 ans révolus - n'était alors pas assez fortuné pour y participer.

59 Sa titularisation comme ingénieur des mines, attaché au Laboratoire de l'École, est bientôt suivie de sa nomination comme secrétaire adjoint de la commission des Annales des mines sous la direction de Dufrénoy, avec la mention «spécialement chargé de la traduction des mémoires étrangers ». II va s'employer à faire renaître les Annales qui, en 1832, inaugurent une nouvelle série.

60 En sus de ses travaux de chimiste, Le Play prépare sa première étude économique fondée sur les statistiques commerciales de l'administration des Douanes (Le Play, 1832). 
du récit de vie de Le Play, notre enquête n'infirme pas ce que l'intéressé a dit de lui-même. Elle le précise et le complète. Car si Le Play pêche de quelque manière dans son récit, c'est moins par des inexactitudes que par des omissions. Parmi celles-ci figurent ses sympathies saint-simoniennes.

\section{UN NON-DIT, LES SYMPATHIES SAINT-SIMONIENNES}

C'est au cours de ces années cruciales qui vont de son admission aux Mines (I827) à sa pleine entrée dans la vie active (I832) et enjambent la Révolution de 1830, que se situe l'épisode saint-simonien de Le Play ${ }^{61}$, sans qu'on puisse le dater avec plus de précision. Cet épisode est préparé par un changement de l'état d'esprit du jeune homme qui devient plus critique à l'égard de la société traditionnelle.

\section{I. UN ENGAGEMENT TARDIF}

Les premiers signes de ce changement sont décelables dans la correspondance qu'il adresse à sa mère, au cours du voyage d'instruction de l'été de I829, évoqué plus haut. Accueilli, au cours de son périple, par divers exploitants, il observe leur mentalité et les juge. D’une famille de propriétaires de la région messine qui exploite des «forges magnifiques» sous la direction d’un gendre ingénieur des mines, il dit: «Cette maison n’est si désagréable que parce que tout le monde est lancé dans un excès de dévotion qui passe les bornes ». Seul le fils échappe à la critique :

C’est un jeune homme charmant qui, malgré les précautions de sa mère, est entré complètement dans les idées du siècle, et n’a pas conservé la moindre trace de jésuitisme. Il a travaillé pour entrer à l'École polytechnique, il a même été reçu avec distinction, mais sa mère n'a jamais voulu lui permettre d'entrer dans ce lieu de perdition, et l'a gardé auprès d'elle, malgré le désir bien naturel qu'il avait d'aller jouir de ses travaux (Le Play, I899, p. 32-33).

61 Remarquons que la chronologie de l'engagement saint-simonien de Le Play coïncide avec la périodisation établie par J.-C. Caron (1991) qui considère le mouvement de la jeunesse parisienne comme «brisé» en |823-1827 avant de connaître un «renouveau» en |828-1830. 
Lanecdote prend tout son sel lorsqu'on découvre, par recoupement ${ }^{62}$, que Le Play est à Hayange, reçu par la famille de Wendel. L'ingénieur des mines, confit en dévotion, qui l'accueille avec tant de froideur, est Théodore de Gargan. Il a épousé Marguerite de Wendel et dirige, parallèlement à ses fonctions d'ingénieur du Corps, l'entreprise familiale. Confirmant l'impression qu'a eue Le Play de ses options politiques, il démissionnera après la révolution de Juillet, se consacrant entièrement à l'entreprise de sa belle-famille. Quant au jeune homme, empêché d’intégrer Polytechnique, «lieu de perdition», c'est son beau-frère, Charles de Wendel qui dirigera seul l'entreprise à la mort de Gargan (I853).

Au cours du même voyage, Le Play découvre les conditions de travail des ouvriers mineurs. De la mine de charbon de Neunkirchen près de Saarbrucken, il dit à sa mère: «J'ai pénétré dans un endroit très proche de la couche embrasée où l'on a une chaleur de plus de $35^{\circ} \mathrm{C}$; les mineurs y ont tellement chaud que, même en étant complètement nus, leur corps est toujours baigné de sueur. C'est, je t'assure, un triste spectacle que celui de ces malheureux quoique le mot soit peut-être impropre, puisqu'en général, les mineurs aiment leur état » (op.cit., p. 36). Plus tard, parvenu à Clausthal, dans le Hartz, il s'indigne face à ces «nuées d'ouvriers qui ont presque tous une santé détruite, qui sont pâles comme des spectres et dont la plupart ont les poignets tordus par l'effet des vapeurs de plomb qui donnent aussi à leurs membres un tremblement continuel et tout cela, pour gagner 7 groschen par jour, c'est-à-dire environ 2I sous » (op. cit., p. 57). En même temps, il les admire pour «leur hardiesse » et la grandeur de leur œuvre. Tout comme l'impressionne l'«admirable organisation (de) l'administration du Hartz $\gg^{63}$.

62 Albert Le Play, éditeur des lettres de son père, a anonymisé les lieux et les personnes. Mais des faits consignés par Le Play permettent de les identifier.

63 Dans son premier mémoire publié par les Annales des mines, Le Play revient sur l'administration des mines du Hartz. En bon ingénieur saint-simonien, il y voit la mise en œuvre du principe d'association dans l'industrie et vante son souci de la condition des ouvriers (Le Play, 1832, p. 518). 
Le Play qui, adolescent, admirait la ferveur religieuse des Vendéens et leur attachement aux Bourbons ${ }^{64}$, a changé. Il est devenu un jeune homme rebuté par la dévotion et le jésuitisme, plein de compassion à l'égard du prolétariat industriel, tout en étant subjugué par les progrès de la métallurgie. «Pour un homme de mon métier», écrit-il à sa mère, «le Hartz est un véritable pays de cocagne». En somme, un futur ingénieur tout disposé à être séduit par le saint-simonisme. De fait, en dépit de son silence sur le sujet, voire de ses dénégations ultérieures, les sympathies saint-simoniennes du jeune Le Play semblent avérées. Un premier indice réside dans une lettre de Jean Reynaud à Euryale Cazeaux où il évoque son réseau proche:

Jamais la nature ne maavait semblé si belle que depuis que je suis saint-simonien, depuis que j'applique tous mes sens à étudier l'œuvre de l'incompréhensible divinité. Au reste, si je puis rester un peu à Bastia, j'espère, comme je le disais à Transon, que la doctrine marchera. C'est presque toujours à lui que j'adresse mes lettres, parce que je ne sais où est Michel, ni où est Le Play (Lettre de Bastia du Io juillet I830 in Reynaud, 1886, p. 113-115). ${ }^{65}$.

Ce dernier fait donc partie, dès cette époque, des apprentis ingénieurs des mines qui connivent à propos du saint-simonisme. Il y contribue par ses premiers travaux personnels. C'est, du moins, ainsi que l'on peut interpréter un article non signé paru dans Le Producteur, gazette saint-simonienne daté du 23 avril I83 I. L’auteur, sous forme d'une correspondance fictive, s’appuie sur l'exemple de l'organisation industrielle du Hartz pour concrétiser ce que serait le monde de la production transformé par le saint-simonisme. Ce texte est plus probablement de Reynaud que de Le Play dont on ne reconnaît pas le style, mais il

64 Après son baccalauréat, Le Play a effectué un stage en Vendée (mai-septembre 1823), aux côtés de l'ingénieur des Ponts et Chaussées, Dan de La Vauterie, chargé de superviser les travaux d'endiguement dans l'île de Noirmoutiers. Ce séjour coïncide avec la visite de la duchesse d'Angoulême, fille de Louis XVI, rescapée de la prison du Temple, qui traverse la région, soulevant l'enthousiasme de la population. Le Play - il a alors dix-sept ans - a été très impressionné par cet évènement.

65 Pour attester de l'affiliation saint-simonienne de Le Play, la correspondance de saint-simoniens notoires comme Reynaud ou Chevalier nous paraît plus probante que les listes publiées ultérieurement par Barthélemy Prosper Enfantin. 
repose sur les observations que tous deux viennent de faire dans le Hartz ${ }^{66}$. Autre indice: lorsque Le Play est titularisé ingénieur ordinaire de $2^{\mathrm{e}}$ classe en octobre I83 I, dans un geste typiquement saint-simonien, il aurait renoncé à sa part de l'héritage familial en faveur de sa sœur. À celle-ci, il aurait déclaré : «À toi tout. Quand un homme a une carrière, il serait un lâche s'il ne suffisait pas à lui-même. » (Mgr Baunard, 1902, p. 284). Mais la preuve la plus tangible de son adhésion saint-simonienne, on la trouve, sous sa plume, dans une lettre du 7 avril $1832^{67}$, adressée à Michel Chevalier, alors directeur du Globe, organe de l'Église, lettre dont les termes sont sans ambiguïté :

Mon cher Chevalier,

Je pars aujourd'hui avec Baudin et Malinvaud ${ }^{68}$ pour faire une petite excursion au Havre et en Normandie ; je vous serais donc bien obligé si vous vouliez avoir la complaisance de dire un mot pour que ma nourriture quotidienne, Le Globe, me soit envoyée au Havre, à partir de demain.

Vous devez bien penser que nous ne manquerons pas de prêcher partout, suivant notre foi.

Malinvaud et Baudin prolongeront la tournée en Bretagne; si vous avez besoin de nous, écrivez-nous, nous vous rendrons tous les services qui pourront dépendre de nous.

Votre tout dévoué camarade.

Le Play

rue aux Cailloux

Ingouville près Le Havre

66 L'auteur voit dans l'exemple du Hartz une préfiguration des «corps industriels tels qu'ils seront institués dans la société Saint-simonienne» (Anonyme, I83I, p. 280).

67 Fonds Enfantin, Bibliothèque de l'Arsenal, Paris.

68 Plus jeune de trois ans, né à Rouen, Désiré Pierre Baudin (1809-1870) est le fils d'un maître de pension. Reçu à Polytechnique en 1826, il a été admis aux Mines en 1828 (AN FI427/3/I). Jean-Firmin Malinvaud (1808-1837), né à Limoges, admis aux Mines lui aussi en 1828, il sera chargé de la chaire de chimie et de métallurgie à l'école des mineurs de Saint-Etienne (1833). II meurt jeune des suites d'un accident du travail (AN FI42732/2). Le Play lui consacre une notice nécrologique dans les Annales des mines, la seule qu'il ait jamais rédigée. 
Sur les prédications annoncées, au Havre et ailleurs, on ne sait rien. Le Play n’en parle aucunement dans son introspection rétrospective. Peut-être un dépouillement de la presse régionale (Journal de Rouen, Journal du Havre), attentive aux évènements locaux, pourrait-il nous renseigner. Mais, durant le printemps de I832, ses colonnes sont surtout occupées par l'épidémie de choléra qui sévit à Paris et se répand en France. Épidémie qui peut aussi expliquer cette «petite excursion » qui éloigne les trois amis des foyers de contamination parisiens...

\subsection{CHEVALIERISTE PLUTÔT QU'ENFANTINISTE}

Quoi qu'il en soit, cette lettre d'adhésion est tout sauf anodine. Elle intervient alors que les saint-simoniens affrontent une double crise. D'une part, ils sont en butte à une répression croissante de la part des autorités depuis leur prise de position en faveur des ouvriers de la soierie de Lyon $^{69}$. D’autre part, ils connaissent de profondes dissensions internes qui aboutissent, à l'automne de I83 I, à la sortie du mouvement de plusieurs de ses membres les plus actifs, suivie de polémiques sans fin affichées sur la place publique. Or, loin de déserter un mouvement qui décline dans un contexte post-révolutionnaire, Le Play affirme, par cette lettre, son appartenance. Mieux, il prend parti et se range du côté de Chevalier lequel est devenu, plus que jamais, le bras droit d'Enfantin ${ }^{70}$. Il ne suit pas son ami Reynaud qui, après sa sortie spectaculaire de l'Église ${ }^{71}$, se livre à une critique en règle des conceptions de ses anciens amis. Au contraire, il reste un lecteur attentif et sympathique de l'évolution ultime du saint-simonisme telle qu'elle s'affirme dans Le Globe dernière manière dont il fait sa «nourriture quotidienne». De janvier à avril $1832^{72}$, Chevalier

69 Voir Rude (1969, p.702-705) où l'auteur détaille l'action de Chevalier et Barrault qui leur valent des poursuites en janvier 1832.

70 Chevalier, déjà responsable de la presse saint-simonienne, a été nommé lieutenant d'Enfantin le 18 février 1832 (Charléty, 1965, p. 120). II assume la nouvelle orientation du Globe devenu pacifiste avec comme sous titre «Appel aux femmes. Organisation pacifique des travailleurs» (ler janvier 1832).

7I Léon Cornudet, alors jeune étudiant en droit, assiste à cette scène historique et en rend compte, le 28 novembre 1831, à son ami Charles de Montalembert, alors en route pour Rome avec Lamennais où ils vont plaider la cause de L'Avenir (Cornudet, 1905, p. 9-12).

72 La dernière parution du journal date du 20 avril 1832. 
y livre, en effet, une série d’articles définissant la «politique nouvelle » saintsimonienne. Cet exposé débute par une réaffirmation du principe de paix internationale comme facteur de progrès («les chances de prospérité sont dans la paix», 3I janvier I832), puis se poursuit par l'idée que les révolutions sont affaire du passé («le génie des révolutions n’a plus à visiter les peuples» 5 février 1832) et qu'il faut aller vers l'association universelle unissant peuples, classes, partis et individus (20 avril 1832). Dans cette perspective, il s'agit, à rebours des projets de conquête ou des missions guerrières soi-disant civilisatrices, de fonder une politique nouvelle qui reconnaisse le «génie propre de chaque peuple» (2 I mars I832) et dépasse les oppositions entre les partis (libéraux, du juste-milieu, légitimistes). Le défi est de «rendre justice à tous les partis et (de) les révéler les uns aux autres par leur élément progressif» (20 avril I832). L'incarnation de cette «politique nouvelle» est le «Système de la Méditerranée» (12 février 1832), vaste «plan de travaux» au service duquel seront affectées les armées transformées en «corps industriel» (8 mars I832). Pour Chevalier, ce programme est une traduction du principe d'association universelle puisqu'il réalisera l'union industrielle et commerciale de l'Occident et de l'Orient (où les peuples ont aussi «soif de progrès », dit-il) et permettra de «régénérer les contrées qui bordent la Méditerranée ». Cette union suppose la construction de voies de communication reliant les grands ports méditerranéens à leur hinterland et au-delà, grâce à un vaste réseau de chemins de fer, «symbole le plus parfait de l'association universelle» (I2 février i832) dont Chevalier brosse le schéma territorial. Cet ensemble d'articles ${ }^{73}$ constitue, en quelque sorte, le testament d'une Église en voie de dissolution. En butte à la répression du pouvoir, connaissant une hémorragie de ses effectifs, l'Église saint-simonienne se voit contrainte de mettre fin, le 20 avril, à la publication de son organe, Le Globe. Ses membres se retirent à Ménilmontant. Bientôt ses chefs seront arrêtés, traînés en justice et condamnés (été-automne I832). Mais Chevalier veut croire que le saint-simonisme a, malgré tout, réussi. Il a ouvert son dernier article par ces mots : «Notre politique est maintenant acquise au siècle. Nos enseignements et prédications, nos missions, nos écrits divers et 
surtout Le Globe... en ont déposé le germe en bien des esprits... D’autres que nous peuvent se charger maintenant d'infiltrer au monde cette politique, et nous sommes sûrs qu'ils le feront, chacun suivant sa nature ».

Tel est donc le couronnement de la pensée saint-simonienne dont Le Play s'imprègne en ce début de i832, alors qu'il entre en fonction à la commission des Annales des mines. Imprégnation passagère? Non car on en retrouve la marque dans sa pensée ultérieure: son attachement à la paix internationale, son rejet des révolutions comme mode de transformation des sociétés, ses réflexions sur les relations commerciales au sein du bassin méditerranéen et son intérêt pour les sociétés de l'Orient, sa volonté de transcender les partis et les classes pour aboutir à une union intellectuelle... Autant d'échos dans son œuvre de la doctrine dessinée par Chevalier, elle-même assez différente de la radicalité d’Enfantin bouleversant les institutions civiles (la famille, l’héritage, etc.).

\subsection{UNE IMPRÉGNATION À LONGUE PORTÉE}

Rétrospectivement, Le Play apparaît comme un de ceux où a été déposé le «germe» saint-simonien (version Chevalier) et qui ont «infiltré au monde cette politique ». Il le fera à sa manière et dans des circonstances auxquelles le grand Michel ne pensait pas encore en I832 comme, par exemple, les expositions universelles, en lien souvent avec des saint-simoniens de l'ère «pratique»: Émile et Isaac Péreire, Paulin Talabot, François Barthélemy Arlès-Dufour et, évidemment, Chevalier lui-même. Le lien avec ce dernier constitue, d'ailleurs, un indice du prolongement de cette influence de jeunesse. Alors que la rupture est définitivement consommée entre Chevalier et Reynaud dès l'automne de ı83 I, Le Play reste en relation suivie avec son aîné, y compris après la dissolution de l'Église saint-simonienne ${ }^{74}$. Premier geste: tandis que Chevalier purge sa peine d'emprisonnement à Sainte-Pélagie, Le Play insère dans les Annales des mines un mémoire qu'il a jadis rédigé. Chevalier en est très touché et le signifie à son camarade qu'il qualifie de «mon cher ami» :

74 On trouve dans le fonds Le Play à la Bibliothèque de l'Institut (Paris), neuf lettres de Chevalier à Le Play, adressées entre 1833 et 1837 dont cinq écrites au cours de la mission américaine de Chevalier. 
Mon frère en venant me voir aujourd'hui m'a apporté la dernière livraison des Annales des mines (septembre-octobre I832). J'ai été agréablement surpris d'y trouver mon mémoire que j'écrivis dans d'autres temps, avec une note flatteuse $^{75}$. Ceci me prouve que [élément ill.] je pense souvent aux bons amis que j’ai laissés dans le corps, aux témoignages d'estime et d'attachement que j’ai reçus de plusieurs des hommes honorables qui en forment la tête ; [...] l'ardeur avec laquelle je suis ma voie présente ne m’empêche pas de fortement tenir à mon passé. Ce souvenir est d'ailleurs parfaitement aujourd'hui; je l'ai accepté comme un souhait de fête, car c'est aujourd'hui que j'ai 27 ans accomplis (Lettre de Chevalier à Le Play à en-tête de «Religion Saint-Simonienne», datée de Sainte-Pélagie, Paris, le Iz janvier I833 [iop.], fonds Le Play, Bibliothèque de l'Institut, Paris - partiellement reproduite in Gallice, 1994).

Et Chevalier fait suivre cet émouvant accusé de réception d'un véritable programme d’action pour un corps des mines dont la mission serait redéfinie. On retrouve ses qualités de prospectiviste, quasiment visionnaire, dans le message qu'il adresse à son interlocuteur et, au-delà, aux ingénieurs :

Vous avez dans vos mains, Messieurs, le combustible et les métaux c'est-àdire deux éléments centraux de toute industrie, de toute fabrication. La vapeur c'est-à-dire le charbon de terre, acquiert une importance qui va croître selon le carré ou le cube du temps, soit comme moteur dans les fabriques, soit comme moteur dans les communications par eau et par terre. Les chemins de fer et les bateaux à vapeur ont un avenir superbe. La machine à vapeur, malgré les ingénieux perfectionnements qu'elle a éprouvés, est encore dans son enfance. [... Les progrès qu'il est aisé de concevoir et d'entrevoir d'ici à dix ans, dans la direction de l'extension de l'emploi des métaux et surtout de la trinité fonte, fer, acier, et sous le rapport des perfectionnements apportés à la machine à vapeur, aux bateaux à vapeur, aux chemins de fer, et aux communications en général, ces progrès, dis-je, sont prodigieux (ibid.).

Les ingénieurs dont Chevalier voudrait qu'ils se mettent à la tête du développement industriel, ont aussi, à ses yeux, des responsabilités vis-à-vis de la classe ouvrière :

75 II s'agit de son étude des mines qui approvisionnent Paris en charbon, notamment celles de Mons (Belgique). Ce travail, déjà publié, est repris dans les Annales des mines en raison «de l'intérêt que présente le mémoire de M. Chevalier» (p. 203, n. I). 
Et puis encore, pour être ingénieur des mines, il n’est pas défendu d’avoir des entrailles. Il y a de superbes questions industrielles qui portent particulièrement sur la condition des classes inférieures, sur les modifications que leur sort a subies, sur les changements introduits dans la vie des peuples par telle ou telle invention (ibid).

Et de conclure sa longue missive par cette phrase: «Vous, en particulier, mon cher Le Play, vous possédez une puissance d’analyse qui vous rendrait de semblables travaux aisés et agréables ».

Cette fidélité à Chevalier n'empêche pas Le Play de rester en relations amicales et intellectuelles avec Reynaud comme l'atteste sa participation, entre I837 et I840, à l'entreprise monumentale de Leroux et Reynaud qu'est l'Encyclopédie nouvelle. Elles culmineront en I 848 lorsque Le Play s'engage très activement aux côtés du sous-secrétaire d'État à l'Instruction publique du Gouvernement provisoire dans la réforme des «hautes études » c'est-à-dire de l'enseignement supérieur. Il est une des chevilles ouvrières de la création de l'École d’administration qui est aussi une machine de guerre contre l'économie politique et... Chevalier. Celui-ci y voit une nouvelle marque de l'hostilité irréductible de Reynaud à son égard. Mais il n'en voudra pas à Le Play dont il défendra, avec une autorité retrouvée, Les Ouvriers européens (1855). Puis, il appuiera la création de la SIEPES que Reynaud, lui, refusera de rejoindre. Désormais, les chemins des deux hommes ne cesseront de se croiser, au Conseil d'État tout d'abord, puis lors des expositions universelles de I855, I862 et I867, également à l'occasion d'enquêtes officielles qu'ils mènent ensemble. Cette collaboration institutionnelle et intellectuelle trouvera une forme d'aboutissement dans une alliance familiale, lorsque Marie Chevalier épouse Albert Le Play en I867.

Le rapport de Le Play aux idées saint-simoniennes et à ses représentants est donc complexe et durable. Il se noue dans l'époque de l'Église pour aller jusqu'à celle du saint-simonisme pratique. La conception sociale que Le Play se forge peu à peu, en conserve la trace, tout en intégrant d'autres influences de jeunesse. Lors de la révolution de I848, à l'économiste britannique George R. Porter, responsable du Board of Trade, il confie : 
À ma sortie des écoles, je me suis trouvé, par exemple, lié d’amitié avec mes contemporains, Michel Chevalier, J. Reynaud, Ch. Duveyrier, Transon, etc. qui s'occupaient alors chaudement des doctrines saint-simoniennes. J'ai suivi plus tard, avec le même intérêt, les travaux des fouriéristes. La conclusion des études assidues que j'ai faites sur toutes ces sectes socialistes: l'idée première est juste à beaucoup d'égard et conforme à celle qui me [mot ill.] moi-même socialiste; mais l'idée définitive, la formule de la doctrine, est toujours fausse. Aussi, tout en restant liés de sympathie avec les écoles socialistes, j’ai toujours refusé de contracter avec elles la moindre liaison et me suis toujours appliqué à ramener à des vues plus justes, plus réelles, ceux de mes amis qui se trouvaient engagés dans les sectes saint-simonienne, fouriériste, etc. (Lettre de Le Play à G.R. Porter, Paris, le 22 avril I848, fonds Le Play, Bibliothèque de l’Institut, Paris).

\section{CONCLUSION Le Play «déromancé »?}

$\mathrm{Au}$ terme de notre enquête - qui appelle d'autres approfondissements -, le portrait de «Le Play en jeune étudiant» apparaît un peu différent de celui qu'il a brossé de lui-même. Le Play n'est plus tout à fait le sage provincial qui a su résister aux tentations idéologiques, se tenant à l'écart des passions de la «génération romantique». Certes, il n’a pas eu l'engagement d'un Chevalier ou d’un Reynaud, ou bien de ses aînés les Buchez et les Bazard. Mais il a bel et bien participé au mouvement de la jeunesse étudiante parisienne. Au regard de son intention de faire la genèse de sa science sociale, sa minimisation des idées saint-simoniennes dans sa formation interroge. Pourquoi, 50 ans après les évènements, taît-t-il encore la place qu'elles ont tenues? Lorsque SaintSimon écrit, dans son Mémoire sur la science de l'Homme (1813), «que ces auteurs [Vicq d'Azyr, Cabanis, Bichat et Condorcet] avaient fait faire un pas bien important à la science, en la traitant par la méthode employée dans les autres sciences d'observation c'est-à-dire en basant leur raisonnement sur des faits observés et discutés, au lieu de suivre la marche adoptée par les sciences conjecturales où on rapporte tous les faits à un raisonnement», n'est-ce pas une déclaration à laquelle Le Play aurait pu souscrire? L'occultation de son saint-simonisme fait partie de son «roman familial» (au sens freudien du terme). Déromancer Le Play, c'est aussi questionner ces non-dits pour en trouver le sens. 


\section{BIBLIOGRAPHIE}

ANONYME, I83I, «Sur l'industrie. Extrait d'une correspondance», L'Organisateur, gazette des saint-simoniens, $n^{\circ}$ 36, $\|^{\mathrm{e}}$ année, avril, p. 277-280.

BAUNARD Mgr, 1902, La Foi et ses victoires dans le siècle présent. Tome II «Frédéric Bastiat, Alexis de Tocqueville, Frédéric Le Play», $7^{e}$ édition, Paris, Librairie Poussielgue.

BELHOSTE B., 200I, «La préparation aux grandes écoles scientifiques au XIX siècle: établissements publics et institutions privées», Histoire de l'éducation, 90, p. I0I-130.

- 2003, La Formation d'une technocratie. L'École polytechnique et ses élèves de la révolution au Second Empire, Paris, Belin.

BEUGNOT J.-Cl. (comte), 1852, Vie de Becquey, ministre d'État et directeur des Ponts et Chaussées et des Mines sous la Restauration, Paris, Firmin-Didot Frères.

BIRE E., 1904, Armand de Pontmartin. Sa vie et ses œuvres 18/1-1890, Paris, Garnier frères.

BOISROUVRAY D. B. (du), 1921, Mgr Gay (1815-1892), évêque d'Anthédon. Sa vie, ses œuvres d'après des documents inédits, Tours, Alfred Mame et fils.

BOUQUET H.-L., I89I, L'Ancien Collège d'Harcourt et le lycée Saint Louis, Paris, Delalain frères.

CARDONI F., 2007, «Précis de la formation d'un ingénieur des mines: Frédéric Le Play de 1806 à 1830 », in A. Savoye et F. Cardoni (dir.), Frédéric Le Play. Parcours, audience, héritage, Paris, Presses de l'École des mines de Paris, p. 13-4I.

CARON J.-Cl., 1991, Générations romantiques. Les étudiants de Paris et le Quartier latin (18/4-184I), Paris, Armand Colin.

CHARLETY S., 1965 (193I), Histoire du saint-simonisme, Paris, Éditions Gonthier.

CHEVALIER M., I832, Religion saint-simonienne. Politique industrielle et système de la Méditerranée, Paris.

CORNUDET L., 1905, Correspondance de Montalembert et de Léon Cornudet 1831-1870, Paris, Honoré Champion.

FLOTTES P., 1927, Pensée politique et sociale d'Alfred de Vigny, Paris, Les Belles Lettres.

FOURCY A., 1828, Histoire de l'École polytechnique, Paris, chez l'auteur à l'École polytechnique. 
GALLICE F., «Les ingénieurs saint-simoniens: le mariage de l'utopie et de la raison?», Recherches contemporaines, 2, 1994, p.5-24.

GOBLOT J.-J., 1995, La Jeune France libérale. «Le Globe» et son groupe littéraire 1824-1830, Paris, Plon.

GRATRY A., 1925 (1874), Souvenirs de ma jeunesse, Paris, Tequi.

ISAMBERT F.-A., 1966, De la charbonnerie au saint-simonisme. Étude sur la jeunesse de Buchez, Paris, Éditions de Minuit.

LEFEBURE DE FOURCY E., 1899 (I882), «Frédéric Le Play. Notice biographique», in F. Le Play., Voyages en Europe 1829-1854, extraits de sa correspondance, publiés par Albert Le Play, Paris, E. Plon, Nourrit et Cie, p. 3-25.

LE PLAY F., 1829, «Analyse d'une tourmaline du Mont Rose», Annales de chimie et de physique, tome 42, p. 270-282.

-, s.d. (c. 1831), Voyage en Normandie et en Bretagne en septembre et octobre 1831, en société avec M. Baumgarten, élève-ingénieur des Ponts et Chaussées, Ms, 14 p., Fonds Le Play, Paris, SESS.

-, 1832, «Observations sur le mouvement commercial des principales substances minérales entre la France et les puissances étrangères pendant les douze dernières années et particulièrement en 1829, 1830 et I83I 》, Annales des mines, II, p.50I-545.

-, 1899, Voyages en Europe 1829-1854, extraits de sa correspondance, publiés par Albert Le Play, Paris, E. Plon, Nourrit et Cie.

-, 1989 (1879), La Méthode sociale, abrégé des Ouvriers européens [...] , Paris, Méridiens Klincksieck.

- et SAINT-LEGER A. (de), 1855, «Mineur de la corporation de mines d'argent et de plomb du Haut-Hartz, Hanovre», in F. Le Play, Les Ouvriers européens, in-folio, Paris, Imprimerie impériale, p. I40-145.

- et SAINT-LEGER A. (de), 1877, «Mineur des corporations de mines d'argent et de plomb du Haut-Hartz, Hanovre», in F. Le Play «Les ouvriers du Nord et leurs essaims de la Baltique et de la Manche», Les Ouvriers européens, deuxième édition, tome III, Tours, Alfred Mame et fils, Paris, Librairie Dentu, p. 99-152.

MONTESQUIEU Ch. (de), 1748, De l'esprit des lois, Genève, Barrillot \& Fils.

REYNAUD J., 1886, Correspondance familière, Paris, Motteroz. 
RUDE F., 1969, L'Insurrection lyonnaise de novembre 1831. Le mouvement ouvrier à Lyon de 1827-1832, Paris, éditions Anthropos.

SAVOYE A. et AUDREN F., 2008, «Les ingénieurs des mines et les sciences sociales émergentes au XIX siècle: le filon leplaysien », in F. Le Play et ses élèves, Naissance de l'ingénieur social, anthologie établie par A. Savoye et F. Audren, Paris, Presses de l'École nationale supérieure des mines, p. 9-22.

SHINN T., 1980, Savoir scientifique et pouvoir social. L'école polytechnique et les polytechniciens, Paris, Presse de la Fondation des sciences politiques.

THUILLIER G., 1962, «Une source documentaire à exploiter: les «voyages métallurgiques» des élèves-ingénieurs des Mines», Annales, XVII-2, p. 302-307. 